\title{
The pattern of reproduction in the mole-rat Heliophobius from Tanzania: do not refrain during the long rains!
}

\author{
M.K. Ngalameno ${ }^{1,2}$, A.D.S. Bastos ${ }^{3}$, G. Mgode $^{2}$ and N.C. Bennett ${ }^{1,4}$ \\ ${ }^{1}$ Department of Zoology \& Entomology, University of Pretoria, Pretoria 0002, South Africa \\ ${ }^{2}$ Sokoine University of Agriculture, P.O.BOX 3000, Morogoro, Tanzania \\ 3Mammal Research Institute, Department of Zoology \& Entomology, University of Pretoria, Pretoria \\ 0002, South Africa \\ ${ }^{4}$ SARChI Chair of Mammal Behavioural Ecology and Physiology, Department of Zoology, University of \\ Pretoria, Pretoria, South Africa
}

\begin{abstract}
The genus Heliophobius comprises of at least six cryptic, topotypical species in the Heliophobius argentocinereus species complex. The current study investigated the breeding patterns of a wildcaught population from Tanzania where the putative species H. emini resides. Individuals were collected on a monthly basis for an entire calendar year. Assessment of foetus presence, gonadal histology, reproductive tract morphometrics in combination with gonadal steroid (plasma progesterone and oestradiol-17 $\beta$ in females and testosterone in males) measurements and field observations revealed that rainfall is important for the onset of breeding. The results further confirmed that breeding is limited to a single, yearly reproductive event synchonised to the long rainfall pattern. The distinct breeding peak in July is associated with an elevation in gonadal mass, increase in concentrations of reproductive hormones and presence of Graafian follicles and corpora lutea in the ovaries of females. These reproductive parameters coincided with the end of the long rainfall period, whereas presence of young in the maternal burrow system corresponded with the start of the short rainfall of East Africa. These findings confirm Heliophobius has a single breeding opportunity each year, and this species is therefore vulnerable to any changes that may impact their climatically attuned breeding patterns.
\end{abstract}

Keywords: Heliophobius, solitary, hormones, histology, precipitation, radioimmunoassay, reproduction, cyt $b$. 


\section{Introduction}

In subterranean mole-rats, breeding is constrained by the ecological conditions and the restrictive nature of the burrow system (Bennett et al. 2000). The subterranean niche prevents the use of many environmental cues used by surface occurring species, for example change in day length (photoperiod) is unlikely to act as a proximate cue because of the slight annual difference in day length. Rainfall on the otherhand is increasingly implicated as a cue for triggering breeding in several solitary subterranean mammals (Dennis and Marsh 1977; Bennett and Faulkes 2000; Šumbera et al. 2003; Herbst et al. 2004; Hart et al. 2006; Katandukila et al. 2013), but a consistent trend is yet to emerge as the importance of this cue varies geographically and across different levels of sociality.

Temperature is another important cue which subterranean mammals may usilise for their activity patterns (Lovegrove, 1989; Šklíba et al. 2007; Lovey et al. 2013; Šklíba et al. 2014; Oosthuizen and Bennett, 2015). The temperatures within the burrow systems of African molerats are generally more muted compared to those experienced above ground, but diurnal and seasonal fluctuations in temperature do occur albeit at lower amplitudes compared to those of above ground conditions (Bennett et al. 1988; Roper et al. 2001; Burda et al. 2007).

In South Africa the mole-rats occurring in winter rainfall regions mate and conceive only during the winter rainfall months, with the young born in the spring when food is widely available and the soil is workable enabling animals to burrow to search for food in the form of underground storage organs of geophytes. Furthemore the moist soil allows dispersal of the young and the establishment of independent burrow systems in three solitary species; the Cape mole-rat, Georychus capensis (Pallas, 1778), the Namaqua mole-rat, Bathyergus janetta (Gmelin, 1788) and the Cape dune mole-rat, Bathyergus suillus (Schreber, 1782) (Bennett and Jarvis 1988; Herbst et al. 2004; Hart et al. 2006). In eastern Africa these reproductive events have been found to coincide with the long and short rainfall periods in the solitary East 
African root rat, Tachyoryctes splendens (Ruppell, 1835), an alloparic and phylogenetically diverse subterranean rodent mole (Katandukila et al. 2013).The solitary silvery mole-rat Heliophobius argentocinereus (Peters 1846) is reported to breed seasonally in Zambia, Kenya and Malawi (Copley 1950, Scharff, 2001, Šumbera et al. 2003). In Malawi breeding has been reported at during the end/or the second half of a rainy season (Šumbera et al. 2003), rather than at the beginning of the rainy season.

All solitary South African species of bathyergid mole-rats studied to date have been reported to be seasonal breeders with their reproduction linked to rainfall (Bennett \& Jarvis 1988; Herbst et al. 2004; Hart et al. 2006). In contrast, the majority of social mole-rats breed throughout the year (Bennett et al. 1988, Sichilima et al. 2008; 2011; Oosthuizen and Bennett 2008). There are, however, two social species which breed seasonally with rainfall; the common mole-rat, Cryptomys hottentotus hottentotus (Lesson, 1812) which occurs sympatrically with the solitary mole-rats Georychus and Bathyergus mole-rat genera (Spinks et al., 1997; 1999), and the highveld mole-rat, Cryptomys hottentotus pretoriae (Roberts, 1913)(van Rensburg et al. 2002, van der Walt et al. 2001).

The Heliophobius argenteocinereus crypic species complex represents an ideal study group for teasing apart variation in environmental drivers of reproduction in subterranean mammals. Members of this taxon are widely distributed throughout eastern, central and southern Africa, and were until recently believed to be monotypic. A phylogeographical study confirming the presence of muliple topotypical species within the complex, allows for a more thorough investigation of reproductive cues. As all species are solitary and share common ancestry, differences in the timing of reproduction will be strongly linked to locality-specific environmental factors. Heliophobius emini (Noack 1894) was selected for this study as it occurs in an area that is geographically and climatically distinct from the single Heliophobius species for which data are available, viz. H. argenteocinereus from Malawi (Šumbera et al. 
2003) but allopatric with another solitary subterranean species T. spendens for which there is extensive reproductive data (Katandukila et al. 2013).

In the absence of nuclear data corrooborating the topotypically discrete mitochondrial lineages (Faulkes et al. 2011) we take a conservative taxonomic approach and refer to the taxon under study as Heliophobius. However, it is pertinent to mention that available chromosome data, although limited, are suggestive that the species identified by Noack (1894) as Heliophobius emini has a $2 n=52$ whereas specimens from eastern Zambia have a $2 n=62$ whilst those from Kenya have a 2n=60 (George 1979, Scharff et al. 2001, Deuve et al. 2007; Faulkes et al. 2011)

By investigating the reproductive biology of Heliophobius from post-mortem examination of gonads and plasma steroids of animals sampled on a monthly basis for a year in Tanzania, as has been done for other subterranean mammals (e.g. van Rensburg et al. 2002; Herbst et al. 2004; Schoeman et al. 2004; Hart et al. 2006; de Bruin et al. 2012, Katandukila et al. 2013), and by recording pregnancies we can determine whether reproductive activity coincides with the rainfall patterns that are characteristic in eastern Africa; namely the long wet season from February to early June and the shorter dry season in September, October and November. The results have important implications for the species in light of changes in climate of equatorial Africa, where rainfall periods shift dramatically inter-annually in concert with El Niño Southern Oscillations, and where temperature and rainfall, have increased and descreased by an average of $0.23^{\circ} \mathrm{C}$ and $3.3 \%$, respectively, per decade since 1960 (McSweeney et al. 2010). 


\section{Materials and Method}

\section{Study site and sampling}

Heliophobius were captured from four main sites in Mvomero District:

$\mathrm{S} 06.82036^{\circ} \mathrm{E} 037.67056^{\circ}, \mathrm{S} 06.97858^{\circ} \mathrm{E} 037.55001^{\circ}, \mathrm{S}^{\circ} 66.98003^{\circ} \mathrm{E} 037.54636^{\circ}$,

S06.98005 ${ }^{\circ}$ E037.54635 in Tanzania. The study was conducted over 12 consecutive months; from January to December 2013. All sites are classified as agri-ecological zones with highly fertile soils supporting a variety of food and cash crops (Morogoro Regional Socio Economic Profile (MRSP)). The study area has two rainy seasons with a mean annual rainfall of 600 $\mathrm{mm}$; the longer period of rainfall (i.e. heavy rainfall) is from March to May with a mean of $470 \mathrm{~mm}$ while a shorter period of rainfall of $130 \mathrm{~mm}$ is from October to December . Dry months are, June, July, August and September (MRSP). June and July months are the transition months between heavy rains and the dry season. Annual average temperature ranges from 18 to $30^{\circ} \mathrm{C}$ with high humidity during March, April and May; as well as October and November (MRSP) (Figure 1).

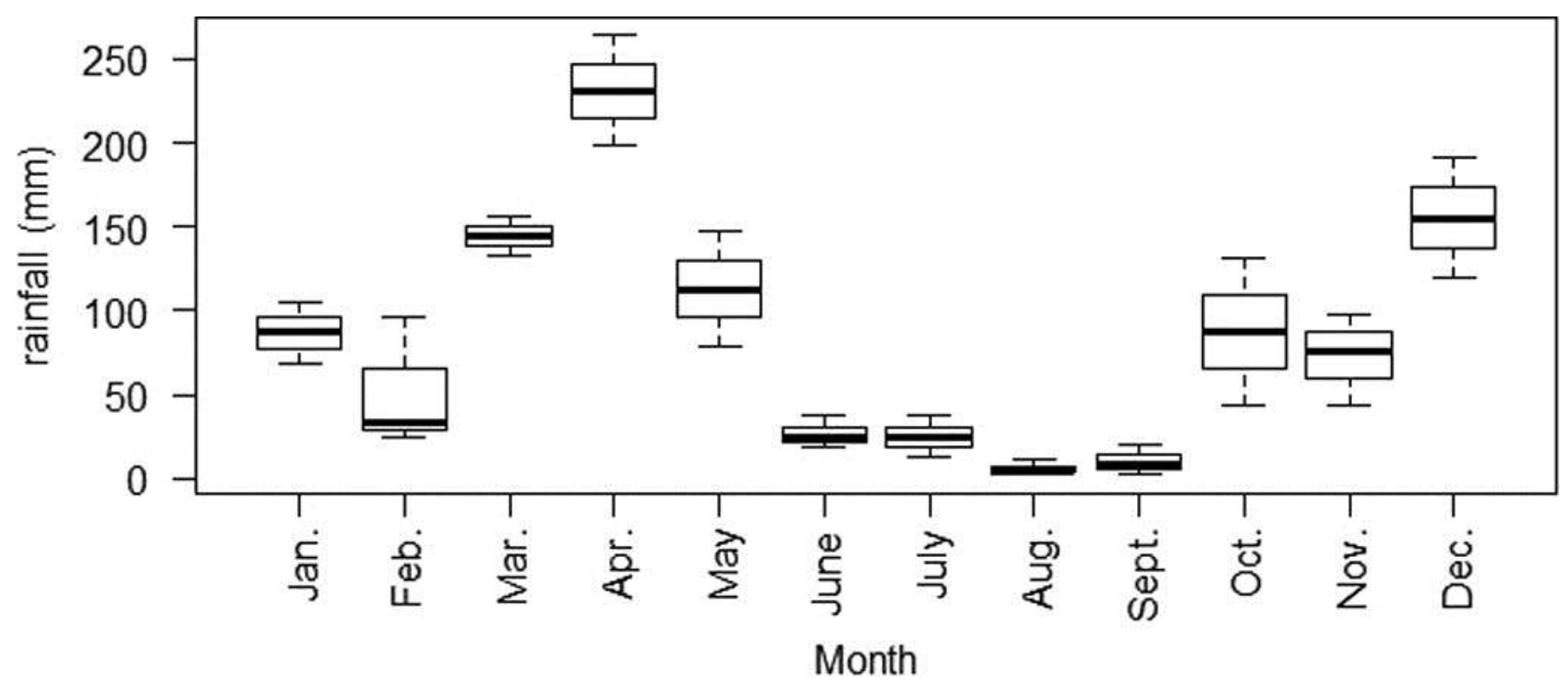

Fig. 1.Mean $( \pm \mathrm{SD})$ monthly rainfall $(\mathrm{mm})$ of Mvomero District, Morogoro, Tanzania, for January until December 2014. Data from Tanzania Meterology Agency. 
Animals were collected from areas subject to widespread crop destruction, where the mole-rat is considered an agricultural pest. Animals were collected using Hickman live traps or by excavation of burrow systems (Hart et al. 2006; Sichilima et al. 2008). Five adult female and five adult male mole-rats were captured monthly for one calendar year resulting in a total of 120 animals (i.e. 60 adult males and 60 adult females). Burrows for capturing animals were selected randomly by opening a distinct burrow system at least $100 \mathrm{~m}$ from one another. The animals were euthanized with an overdose of chloroform and the body mass subsequently recorded. Burrow entrances were left open to check for subsequent blocking and if arising traps were inserted (multiple occupancy usually resulted when there was a mother and offspring).

The presence or absence of elongated teats and/ or a perforate/imperforate vagina were recorded in females. Blood was drawn from the heart of the sacrificed animal using heparin coated syringes and centrifuged at 500g (3000 rpm) for 10 minutes. Following centrifugation plasma was removed using a pipette, transferred to a new tube and stored at $-20{ }^{\circ} \mathrm{C}$. Gonads (testes/ovaries) were removed and fixed in Bouin's fixative for a period of $18 \mathrm{~h}$ prior to being stored in $70 \%$ ethanol. The reproductive status of females was further assessed by recording the presence or absence of embryos/foetuses following the opening of the abdominal cavity. Lactating females could be identified by the presence of prominent teats which released milk on squeezing and / or the occupancy of young, either juvenile(s) or infant(s) in their burrows. Juveniles and sub-adults of both sexes co-habiting their mother's burrow were recorded on capture to assess recruitment to the population of Heliophobius from reproductive animals collected.

The mass (g) of the gonads was measured using a Sartorius scale (Zeiss, Germany). Following weighing the gonads were stored in picric acid for $24 \mathrm{~h}$ and subsequently stored in $70 \%$ ehtanol until being used for histological preparation. The gonads were then sequencially 
dehydrated by placing them in containers of increasing alcohol concentration and subesquently embedded in a block of paraffin wax before sectioning at a thickness of $7 \mu \mathrm{m}$ using a rotary microtome (820 Spencer, American Optical, Scientific Instrument Division, Buffalo, New York, U.S.A.). Sections were mounted on microscopic slides after being dipped in water at $45^{\circ} \mathrm{C}$ mixed with gelatine as an adhesive. The mounted sections were subsequently dried in an oven at $36{ }^{\circ} \mathrm{C}$ for a period of $72 \mathrm{hrs}$ and then stained with Ehrlich's haematoxylin and counter-stained with eosin (Drury \& Wallington 1967). The stained sections were covered by slide cover slips secured with resin solution (Microscopy Entellan glue, Germany) and dried in an oven at $36{ }^{\circ} \mathrm{C}$ for $48 \mathrm{hrs}$.

\section{Testicular histology}

Thirty randomly selected sections taken from the mid region of the testes were used to estimate the mean diameter of seminiferous tubules with a light microscope (Diaplan, Ernst LeitzWetzlar GmbH, Germany). Seminiferous tubules were photographed at 10x magnification using a digital camera attached to a microscope (Moticam 1000 1.3 M Pixel USB 2.0, Motic China Group, LTD., Xiamen, China). The diameters ( $\mu \mathrm{m})$ of 1800 seminiferous tubules were measured using Motic Images Plus 2.0ML (Motic China Group, Ltd., Xiamen, China). The larger diameter of the seminiferous tubules implies an active testes with greater sperm production.

\section{Ovarian histology}

The sectioned ovaries were examined under a light microscope for the numbers of primordial, primary, secondary, tertiary and Graafian follicles as well as corpora lutea following Bloom \& Fawcett (1962), van Rensburg et al. (2002) and Hart et al. (2006). 100×, 200× and 400×magnifications were used consecutively (van Rensburg et al. 2002; Hart et al. 2006). The follicles of each category for each section were counted. Counting error was taken into 
account using the method of Borgeest et al. (2004). The sections were photographed using a digital camera attached to a light microscope (Moticam 1000 1.3 M Pixel USB 2.0, Motic China Group, LTD., Xiamen, China).

\section{Radioimmunoassay}

\section{Progesterone}

Plasma progesterone concentrations were measured using a commercially available kit (Coata-Count MG12171, IBL International GmBH, Hamburg, Germany). The assay enabled the determination of testosterone concentrations over the range 6 to $1200 \mathrm{ng} / \mathrm{dl}$. The crossreactivity of the antibody with other naturally occurring steroids was $<0.1 \%$ except for $5 \alpha$ Pregnan-3, 20-dione (3.4\%), 17 $\alpha$-Hydroxyprogesterone (1.5\%), 20- $\beta$-dihydroprogesterone (3.2\%) and 11-Deoxycorticosterone (0.3\%). A plasma sample with a high concentration of progesterone was double diluted using the assay buffer as a matrix 1:1 to 1:8 then assayed. The slopes of serial double dilution and standard curve were compared to check for parallelism (ANCOVA: $F=5.26, P=0.07, n=4$ ) following a log-logit transformation of the data (Chard 1987). The intra-assay coefficient of variation for the plasma pool was $6.7 \%$ and sensitivity of the assay was $1.35 \mathrm{nmol} / \mathrm{L}$.

\section{Oestradiol $-17 \beta$}

Oestradiol -17ß assays were performed as described by Herbst et al. (2004) using the Coat-aCount MG12101oestradiol kit (IBL International GmBH, Hamburg, Germany). The crossreactivity of the antibody to all naturally occurring steroids was $10 \%$ with oestrone, $<5 \%$ with oestriol, oestrone- $\beta$-D-glucoronide, oestone-3-sulphate, d-equilenin, 17 $\beta$-oestradiol-3monosulphate, testosterone and androsterone. The assay was validated for plasma of $H$. emini by testing the slope of the curve produced using serial dilutions of un-extracted mole-rat plasma obtained from a female with high oestradiol concentrations (over the range 1:1 to 
1:32) against the standard curve. After logit-log transformation of the data (Chard 1987), slopes of the lines were compared and found not to differ significantly from the reference preparation (ANCOVA, $F=4.4, P=0.09, n=4$ ). The intra-assay coefficient of variation for repeated determinations of a quality control was $8.3 \%$ and sensitivity of the assay was $10 \mathrm{pg} / \mathrm{ml}$.

\section{Testosterone assays}

The plasma testosterone concentrations were measured using the commercially available Coat-a-Count MG12191 testostrone kit (IBL International GmBH, Hamburg, Germany). The assay was able to determine testosterone concentrations of 6 to $1200 \mathrm{ng} / \mathrm{dl}$. Cross reactivity of the Coat-a-Count testosterone antibody was $1.8 \%$ with 19 -Nortestosterone, $0.31 \%$ with dihydrotestosterone and less than $0.01 \%$ with other steroids tested. We validated the assay by testing for parallelism between serial dilutions of silvery mole-rat plasma (obtained from an individual with high testosterone concentrations) and the standard curve (Chard 1987). The curves were parallel and not significantly different from the reference preparation (ANCOVA, $F=1.8, P=0.2, n=6)$. The intra-assay coefficient of variation was $7.6 \%$. The sensitivity of the assay (90\% binding) was $5 \mathrm{ng} / \mathrm{dl}$ or $0.175 \mathrm{nmols} / \mathrm{l}$.

Descriptive and statistical analyses were performed using R i386 3.2.2 and Excel 2007. The relationship between variables such as gonadal mass, volumes and rainfall were evaluated using correlation statistical analyses. The normality of the data within each month was tested using the Shapiro-Wilk normality test (Lilliefors 1967) as a consequence parametric tests were used in subsequent analyses. Annual variation in hormone concentrations and gonadal metrics was investigated using ANOVA with Tukey's honest significance difference (HSD) to evaluate significant differences. All descriptive results in the figures are presented as mean \pm SD (standard deviation of mean). 


\section{Species designation}

Clade and species assignment was determined by cyt $b$ gene amplication and nucleotide sequencing of 10 randomly selected individuals ( 5 males and 5 females), using previously described primers and thermal cycling conditions (Bastos et al. 2011). Full-length gene sequences submitted to Genbank under accession numbers KX060579-KX060580, were complemented with homologous data from two prior studies (Faulkes et al. 2004 and 2011) and the best-fit model of sequence evolution identified under the $\mathrm{AIC}_{\mathrm{C}}$ in Mega5 (Tamura et al. 2011) was subesequently used for maximum likelihood inferences.

Two haplotypes were recovered from the 10 individuals selected at random for genetic characerisation. One of these had $100 \%$ nucleotide sequence identity to AY425935 and AY425938, which correspond to clade 3 Heliophobius museum voucher QM1026 and QM1023, respectively and the other represents a new haplotype that differs from the aforementioned reference strains at nucleotide position $604(\mathrm{G} / \mathrm{A})$. This first base position mutation results in a non-synonymous amino acid substitution at position 202 (Ala/Thr). The two haplotype sequences generated in this study cluster within the $H$. emini clade defined by Faulkes et al. (2011), with 100\% bootstrap support (not shown).

\section{Results}

\section{Testicular metrics and histology}

The testicular mass increased over the period February to March and decreased from July to August and remained reduced until January (Figure 2A). The mass of the testes was significantly different between months; $F_{(11,48)}=4.811, n=60, P<0.0001$, with the months of the long wet season having males with heavier testes per gram mass of animal than those of the dry months and the short rains of October and November $\left(F_{(1,58)}=15.58, n=60\right.$, 
$P<0.0002$ ) (Figure 2B). Significant differences were clearly observed between June and

August $(\mathrm{p}=0.012)$, June and December $(\mathrm{p}=0.005)$, March and December $(\mathrm{p}=0.02)$, May and

December $(p=0.033)$, October and June $(p=0.008)$ and October and May $(p=0.04)$. Testicular mass of the breeding and non breeding animals was not influenced by body mass; $\mathrm{F}(56,3)=$ $2.033, \mathrm{n}=60, \mathrm{p}=0.16)$.

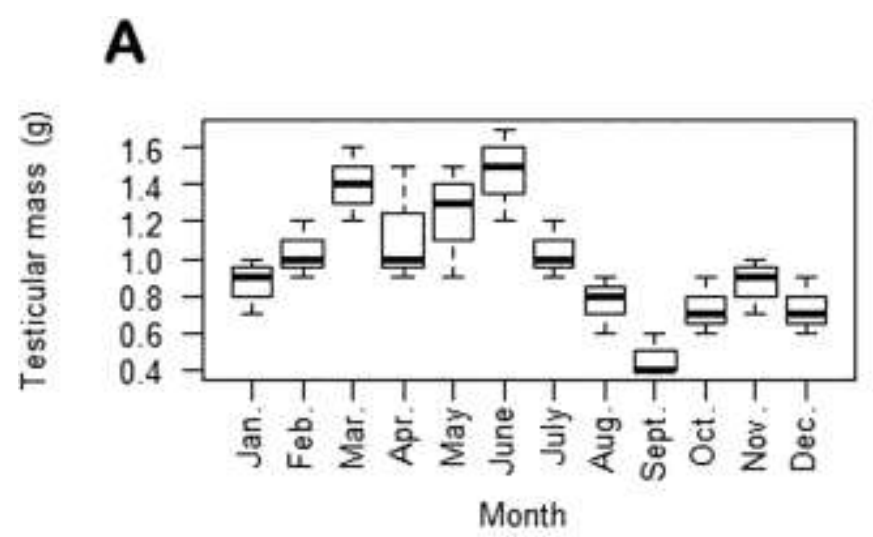

B
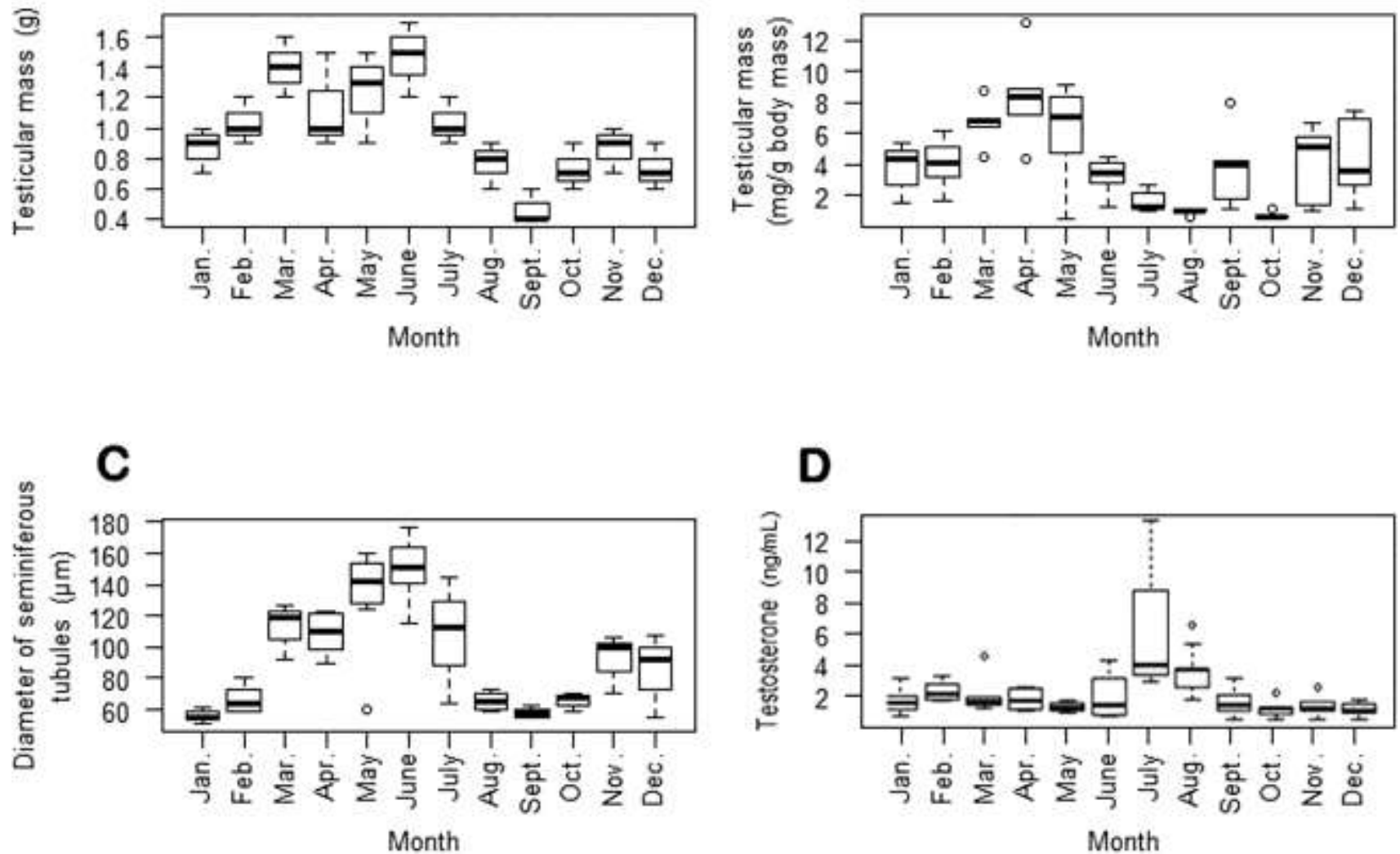

Fig. 2.Monthly fluctuations (mean \pm SD) for raw testicular mass $(A)$, testicular mass milligrams per gram body mass (B), diameter of seminiferous tubule (C), and plasma testosterone concentration (D) in Emin's mole-rat (Heliophobius argenteocinereus emini) from January until December 2014.

The mean diameter of the seminiferous tubules was greatest in May, June and July, whereas the lowest mean diameter was in August, September and October and again in January (Figure 2C). Variation in the diameter of seminiferous tubules between months was significant; $\left(F_{(11,1788)}=115.9, n=1800, P<0.0001\right)$ with breeding months (the long rains) showing males with increased diameter of seminiferous tubules than non-breeding months 
(dry months and short rains) $\left(F_{(1,1328)}=390.9, n=1330, P<0.0001\right)$. Significant differences were observed between; September and April, October and April, June and August, March and August, May and August, June and December, March and December, May and December as well as May and February $(\mathrm{P}<0.0001)$. The width of the seminiferous tubules was positively correlated with the peak of rainfall (Pearson's correlation; $r=0.123, n=1799, P$ $<0.0001)$.

\section{Ovarian metrics and histology}

The mass of the ovaries of females were greatest in March, April and May, but remained relatively small for the rest of the year (Figure 3A). Ovarian mass varied between breeding and non-breeding months $\left(F_{(1,58)}=82.91, n=60, P<0.0001\right)$ (Figure 3B). Significant differences were observed between; August an April, December and April, November and April, March and December, May and January and May and July $(\mathrm{P}<0.0001)$.

The follicular development varied over the year, and as expected, primordial follicles were uniform and showed no clear pattern, but the highest numbers were recorded in March through to July (Table 1). Primary follicles increased from June to March increasing dramatically in April and then decreased, reaching the lowest number in October (Table 1). The numbers increased almost three-fold in November and then decreased to almost by half the amount in December (Table 1). The number of secondary follicles increased in January, peaking in June, then decreased gradually through to September. The numbers increased again in October and November and finally dropped off again in December (Table 1). Tertiary follicles increased sharply from January to April, slightly decreased in May and gradually decreased in November whereafter they increased sharply (Table 1). Graffian follicles increased in number from January reaching a peak in April. The number decreased gradually from May to October, increased in November and decreased again in December (Table 1). The number of corpora lutea increased gradually from February to May, followed by a sharp 
increase in June and July. The numbers decreased to zero in September then increased marginally from October to December (Figure 3C).

Table 1: Mean \pm SD of follicular types found in ovary over a single calendar year

\begin{tabular}{|c|c|c|c|c|c|}
\hline \multirow[t]{2}{*}{ Month } & \multicolumn{5}{|c|}{ Follicular cells } \\
\hline & Primordial & Primary & Secondary & Tertiary & Graffian \\
\hline January & $19 \pm 2.14$ & $0.38 \pm 0.0$ & $0.23 \pm 0.43$ & $0.15 \pm 0.37$ & $0.08 \pm 2.7$ \\
\hline February & $18.75 \pm 2.06$ & $0.25 \pm 0.5$ & $0.25 \pm 0.5$ & $0.5 \pm 0.57$ & $0.25 \pm 0.5$ \\
\hline March & $20 \pm 3.29$ & $0.67 \pm 0.54$ & $0.33 \pm 0.51$ & $0.5 \pm 0.54$ & $0.5 \pm 0.54$ \\
\hline April & $18.4 \pm 2.7$ & $0.8 \pm 0.48$ & $0.4 \pm 0.54$ & $0.6 \pm 0.54$ & $0.8 \pm 0.44$ \\
\hline May & $19.4 \pm 2.44$ & $0.57 \pm 0.53$ & $0.43 \pm 0.53$ & $0.57 \pm 0.53$ & $0.57 \pm 0.53$ \\
\hline June & $19.4 \pm 2.38$ & $0.25 \pm 0.5$ & $0.5 \pm 0.5$ & $0.25 \pm 0.5$ & $0.5 \pm 0.57$ \\
\hline July & $19 \pm 1.63$ & $0.25 \pm 0.5$ & $0.25 \pm 0.5$ & $0.25 \pm 0.5$ & $0.25 \pm 0.5$ \\
\hline August & $15 \pm 4.47$ & $0.33 \pm 0.5$ & $0.22 \pm 0.44$ & $0.11 \pm 0.33$ & $0.11 \pm 0.33$ \\
\hline September & $18.5 \pm 2.73$ & $0.25 \pm 0.46$ & $0.25 \pm 0.46$ & $0.13 \pm 0.35$ & $0.13 \pm 0.35$ \\
\hline October & $14.6 \pm 3.78$ & $0.2 \pm 0.44$ & $0.4 \pm 0.54$ & $0.2 \pm 0.44$ & $0.2 \pm 0.44$ \\
\hline November & $18.2 \pm 2.59$ & $0.6 \pm 0.54$ & $0.4 \pm 0.54$ & $0.6 \pm 0.54$ & $0.4 \pm 0.54$ \\
\hline December & $12.67 \pm 2.5$ & $0.33+0.49$ & $0.17 \pm 0.4$ & $0.17+0.4$ & $0.33 \pm 0.51$ \\
\hline
\end{tabular}



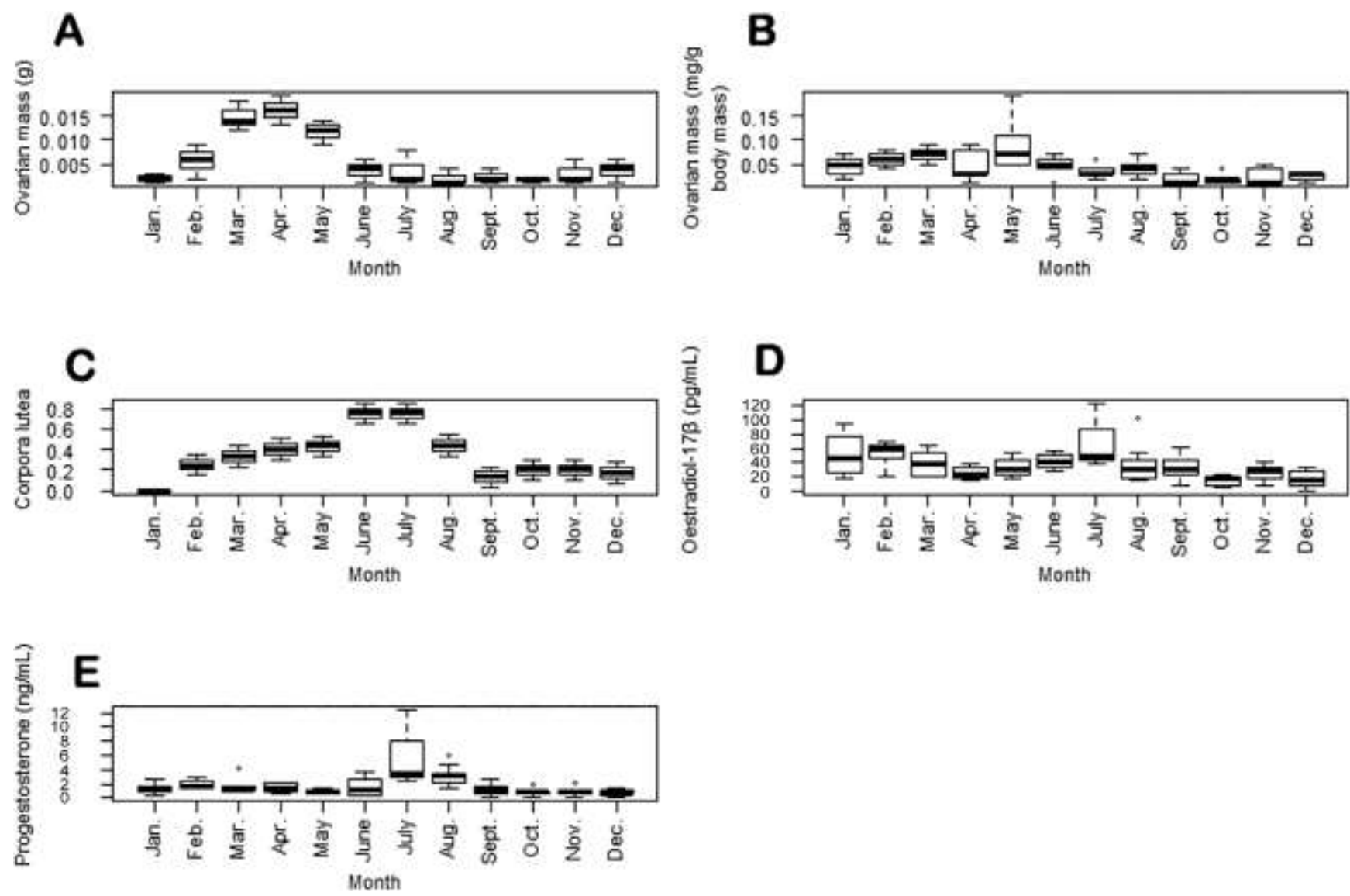

Fig. 3.Monthly fluctuations (mean \pm SD) for raw ovarian mass $(A)$, ovarian mass milligrams per gram body mass (B), number of corpora lutea (C), plasma œstradiol-17 $\beta$ concentration (D), and plasma progesterone concentration (E) in Emin's mole-rat (Heliophobius argenteocinereus emini) from January until December 2014.

\section{Hormonal profiles}

The concentration of testosterone in the males was generally low throughout the year, but increased in April and peaked in June thereafter the concentrations dropped through to March (Figure 2D) . The concentration of testosterone varied between months $\left(F_{(11,46)}=3.212, n=58\right.$, $P=0.00261)$.

The concentration of testosterone in male mole-rats increased during the long rains and was lowest during the dry season with maxima coinciding with peaks in precipitation (see figures 1 and 2D). 
Levels of oestradiol increased from April, peaking in July whereafter there was a gradual decline through to October, with a slight increase observed in November and December (Figure 3D). Progesterone was high in June, July and August then declined from September, remaining very low for all other months (Figure 3E). There was a significant positive correlation between progesterone concentration and number of corpora lutea $(r=0.42, n=75$, $P<0.0002)$.

\section{Pregnancies and lactation}

A total of 120 mole rats were sampled (60 males and 60 females). Out of 60 sampled female animals, $12(20 \%)$ were pregnant and $13(21.7 \%)$ were lactating. Pregnancies were observed initially in June when two of five females (40\%) were pregnant. The percentage increased in July when all five female animals sampled (100\%) were pregnant. Pregnancies decreased in the following months, whereby three females $(60 \%)$ and two females $(40 \%)$ were found to be pregnant in August and September respectively. No pregnancies were observed after September or before June. This is reflected in the number of corpora lutea, which increased from March to May (these are probably corpora lutea of ovulation). In June and July there was peak in numbers of corpora lutea, the corpora lutea of pregnancy, which dropped gradually in August and reached the lowest number from September through to February (Figure 3C).

A total of $50 \%$ of pregnant females were found to have five embryos, three on the left uterine horn and two on the right, $35 \%$ had six, three on either uterine horn and $15 \%$ were found to have five embryos four on the left horn and one on the right. Based on the number of embryos the mean litter size of Heliophobius from Tanzania was 5.3 \pm 0.5. However the number of pups found in maternal burrows was lower with a the mean litter size of $4.4 \pm 0.5$. Lactating animals were first observed in September in which four of the five females were lactating (80\%). All female animals sampled in October and November were lactating and this 
decreased dramatically in December, whereby only one out of five sampled females were found to be lactating $(20 \%)$.

\section{Presence of juveniles.}

A total of 47 juveniles (20 male and 27 female) were sampled in maternal burrow systems. Juveniles were captured in the months of September, October and November. The presence of juveniles coincided with the short rainfall. We predict that in the months of December to February that these individuals would disperse to establish their own independent burrow systems dispersing off from the maternal burrow system.

\section{Discussion}

A field-based study based on post-mortem examination of animals using a number of reproductive indices in Heliophobius from Tanzania showed a single clear breeding period. The reproductive tract morphometrics, gonadal histology and measurement of circulating gonadal steroids revealed one single distinct breeding period that coincided with the precipitation pattern of the long rains in Tanzania. Oestradiol $17 \beta$ and the presence of Graafian follicles in April and May imply that the females ovulate during the height of the long wet season. Likewise, male Heliophobius also showed peaks in testosterone around April and June, had larger seminiferous tubules and heavier testicular mass around April to June. These findings suggest that the adult males and females prepare for mating during the period of April to June. The mating period may reflect a time when the sexes are more tolerant of one another to promote copulation and ensure maximal reproductive success, but is also a period when increased territoriality between same-sex individuals may arise, as has been found in other subterranean mammals during the breeding season (Bennett and Jarvis, 1988; Bennett et al. 1991). It is unknown if the sexes use seismic communication as has been 
reported for other solitary bathyergids (Bennett and Jarvis 1988; Narins et al. 1992; Herbst et al. 2004; Hart et al. 2006) and spalacids (Heth et al., 2007; Rado et al. 1987; Hrouzkova et al. 2013; Katandukila et al. 2013).

The sexes synchronise the maturation of their reproductive organs and the sperm and ova to maximise conception before the end of the wet months. The rainfall results in the soil becoming more workable so that excavation and digging can arise and hence the sexes pair up, however, we currently have little information of how this arises in the field.

Patzenhauerova et al. (2010) using microsatellites report Heliophobius argenteocinereus to exhibit polygyny. The population they studied was found to have a female biased sex ratio. Due to the large distances between burrow systems it is speculated that males might seek females aboveground.

The presence of numerous corpora lutea of pregnancy, higher circulating progesterone concentrations and presence of embryos suggest that maximum pregnancies occur in June and July, falling off in August, following the long rains. These pregnancies probably reflect the optimal period when mating could arise and when constraints on burrowing are minimal as has been suggested for other subterranean mammals (Emlin and Oring 1977; Isaac and Johnson 2003). Lactation was first reported in September, but was frequent in October and November, this coincides with the short rainfall period. As a consequence the soils would be soft and workable during this time and would allow lactating females with young to access the geophytes and grass rhizomes and thus enable these mothers to care for the juveniles over this period. The span of first pregnancy to first lactation and maximal pregnancy to maximal lactation is around three months, which fits with the estimated gestation of 87 days as reported by Jarvis (1969) and Šumbera et al. (2003) for Heliophobius from Kenya and Malawi, respectively. By breeding seasonally animals can time their reproduction in order 
that the pups are born at a point which maximises offspring survival in the environment (Ims 1990).

Many organisms use environmental proximate cues to trigger the onset of reproductive activity. In this study it would appear that the onset of the long rains triggered reproductive activity. In contrast to the East African root rat, mating did not take place early in the rains, but moreover during the latter part of the long rains. The role of photoperiod in subterranean rodents appears to be relatively unimportant. In the spalacid, Spalax ehrenbergi (Nehring 1898) photoperiod has a role in thermoregulation (Haim et al. 1983), similarly several species of bathyergid are able to entrain their locomotory activity patterns to different lighting schedules (Oosthuizen et al. 2003; Hart et al. 2004; de Vries et al. 2008). However, rainfall is undoubtedly the most important environmental cue acting on subterranean mammals in that it can be detected underground because it softens soil and also brings about plant growth and flushes of vegetation (Dennis \& Marsh 1997). Indeed, all solitary South African bathyergid mole-rats studied to date gauge their breeding events with extended periods of rainfall (Bennett and Jarvis 1988; Herbst et al. 2004; Hart et al. 2006). The results of our study are consistent with those for Heliophobius from Malawi (Šumbera et al. 2003) in that reproduction arises in the latter part of the long rains.

The finding of a single litter born per annum per adult female corresponds well with the findings of Copley (1950) and Šumbera et al. (2003) who found a single litter to be produced during the long rains in Kenya and Malawi respectively. The litter size of Heliophobius from Tanzania was higher compared to $3.2 \pm 0.9$ observed in Heliophobius from Malawi (Sumbera et al 2008). Birthing of pups during periods of good rainfall ensures the acquisition of quality food resources for lactating females and their young, as the soil is softened and burrowing is made more efficient. Precipitation is important for the re-sprouting, regeneration and an overall increase in plants succession and vegetation cover (Bennie 1991; Pregitzer and King, 
2005). Juvenile and/or sub-adults were found in the maternal burrow system during the months of the short rains and dispersal of sub-adult mole-rats probably occurs from December onwards as no juveniles were caught with their mothers after this time. This observation implies that the timing of dispersal of sub-adult mole-rats from their natal burrow systems occurs prior to the onset of the next long rains to ensure that the offspring are fit enough and have access to maximal vegetation and food resources for independence when setting up their own tunnel system. This breeding strategy and the associated dispersal from natal burrows is documented for other solitary subterranean rodents from South Africa (e.g Georychus capensis: Jarvis \& Bennett, 1991, Bathyergus species: Bennett and Faulkes 2000; Herbst et al. 2004). The dispersal of sub-adults during periods of rain when food is plentiful (Rado et al. 1992; Le Galliard et al. 2012) and the soil is workable (Williams and Cameron 1984) enables sub-adult Heliophobius to successfully establish their own independent burrow system (Jarvis and Sale 1971; Bennett et al. 1991; Herbst et al. 2004).

Our findings from the reproductive hormone profiles, histological assessment of gonadal characteristics and field observations have clearly revealed that this population of Heliophobius is a strictly seasonal breeder having a single period of reproductive activity within a year. Heliophobius from Tanzania shows peaks of reproductive hormone concentrations, pregnancies, births and sub-adult dispersals concomitant with peaks of precipitation. This suggests that precipitation is the key factor which triggers their seasonal reproduction. The long gestation period precludes the production of a second litter during the short rains. In contrast, the East African root rat is capable of such fecundity due to the much shorter gestation period of around 38 days (Katandukila et al. 2013). The restriction of reproduction to a single event a year makes this species susceptable to climate instability as a result of the action of El Niño and El Niña weather oscillations in the Indian ocean. These 
weather phenomena may bring about either higher and lower rainfall in regions of Tanzania occupied by Heliophobius (McSweeney et al. 2010).

Additional studies on other members of the Heliophobius species complex are required to understand how reproduction varies between species and localities. As these species are cryptic it is important that these initiatives are conducted within a phylogeographical framework.

\section{Acknowledgements}

MKN was sponsored by a student bursary from DAAD in Germany. We thank the Tanzania Commission for Science and Technology (COSTECH) and Ministry of Natural Resources and Tourism Tanzania for granting research permit and export permit for gonads and plasma of mole-rats to the University of Pretoria. We acknowledge import permits from DAFF (13/1/1/30/0-2015/05/005641) and Gauteng Nature Conservation (CPB6-2386) and an export permit from the Ministry of livestock and fisheries development (68368). NCB acknowledges funding from the South African DST/NRF SARChI Chair for Mammal Behavioural Ecology and Physiology, University of Pretoria.

\section{Literature cited}

Bastos, A.D.S., Nair, D., Taylor, P.J., Brettschneider, H., Kirsten, F., Mostert, M.E., von Maltitz, E., Lamb, J.M., van Hooft, P., Belmain, S.R., Contrafatto, G., Downs, S. and Chimimba, C.T. 2011. Genetic monitoring detects an overlooked cryptic species and reveals the diversity and distribution of three invasive Rattus congeners in South Africa. BMC Genet. 12: 26.

Bennett, N.C. and Jarvis, J.U.M. 1988. The reproductive biology of the Cape mole-rat, Georychus capensis (Rodentia: Bathyergidae). J. Zool. (Lond.). 214: 95-106. 
Bennett, N.C., Jarvis, J.U.M. \& Davies, K.C. 1988. Daily and seasonal temperatures in the burrows of African rodent moles. S. Afr. J. Zool. 23: 189-196.

Bennett, N.C., Jarvis, J.U.M., Aguilar, G. H. and McDaid, E.J. 1991. Growth and development in six species of African mole-rats (Rodentia: Bathyergidae). J. Zool. (Lond.). 225: 13--26.

Bennett, N.C., Jarvis, J.U.M., Millar, R.P., Sasano, H. and Ntshinga, K.V. 1994. Reproductive suppression in eusocial Cryptomys damarensis: Colonies social-induced infertility in females. J. Zool. (Lond.). 233: 617-630.

Bennett, N. C. and Faulkes., C.G. 2000. African Mole-rats: Ecology and Eusociality. Cambridge University Press, Cambridge, U.K.

Bennett, N.C., Faulkes, C.G. and Molteno, A.J. 2000. Reproduction in subterranean rodents. In Life underground, the biology of subterranean rodents: 145-177. Cameron, G.N., Lacey, E.A. \& Patton, J. (Eds). Chicago: University of Chicago Press.

Burda, H, Šumbera, R and Begall, S. 2007. Microclimate in Burrows of Sunbterranean rodents - revisited. Subterranean rodents: News from underground 21-33. Begall, S., Burda, H. \& Schleich, C.E. Spinger-Verlag Berlin Heidelberg

Bennie, A. T. P. 1991. Growth and mechanical impedance. Plant roots: the hidden half. Marcel Dekker, New York. pp 393-414.

Bloom, W. And Fawcett, D. W. 1962. A textbook of histology. 8th ed. WB Saunders Co., Philadelphia.

Borgeest, C., Miller, K. P., Gupta, R., Greenfeld, C., Hrushka, K.S, Hoyer, P. and Flaws, J.A. 2004. Methoxychlor-induced atresia in the mouse involves Bcl-2 family members, but not gonadotropins or estradiol. Biol. Reprod. 70: 1828-1835.

Chard, T. 1987. An introduction to radioimmunoassay and related techniques. Amsterdam Elsevier, The Netherlands.

Copley, H. 1950. Small mammals of Kenya. Highway Press, Nairobi, Kenya.

De Bruin, P. R., Viljoen, H., Sichilima, A.M. and Bennett, N.C. 2012. Socially induced infertility in Ansell's mole-rat: are there depressed hormone levels in non-reproductive males and females? J. Zool. (Lond.). 286: 15-21. 
Deuve, J.L., Bennett, N.C., Britton-Davidian, J. \& Robinson, T.J. (2008). Chromosomal phylogeny and evolution of the African mole-rats (Bathyergidae). Chromosome Res.16: 57-74.

De Vries, J.L., Oosthuizen, M.K., Sichilima, A.M. and Bennett, N.C. 2008. Circadian rhythms of locomotor activity in Ansell's mole-rat: are mole-rat's clocks ticking? J. Zool. (Lond.). 276: 343-349.

Dennis, A.J. \& Marsh, H. 1997. Seasonal reproduction in musky rat-kangeroos, Hypsiprymnodon moschatus: a response to changes in resource availability. Wildl. Res. 24: 561-578.

Drury, R.A.B. and Wallington., E.A. 1967. Carleton's histological technique. Oxford University Press, New York.

Emlen, S. T. and Oring, L.W. 1977. Ecology, sexual selection, and the evolution of mating systems. Science 197: 215-223.

Faulkes, C.G., Verheyen, E., Verheyen, W., Jarvis, J.U.M. and Bennett, N.C. 2004. Phylogeographical patterns of speciation and genetic divergence in African mole-rats (Family Bathyergidae). Mol. Ecol. 13, 613-629.

Faulkes, C.G., Bennett, N.C., Cotterill, F.P.D., Stanley W., Mgode, G.F. and Verheyen, E. 2011. Phylogeography and cryptic diversity of the solitary-dwelling silvery mole-rat, genus Heliophophobius (family: Bathyergidae). J. Zool. (Lond.). 285: 324-338.

George, W. 1979. Conservatism in the karyotypes of two African mole-rats (Rodentia, Bathyergidae). Z. Saugetierkunde 44: 278-285.

Haim, A., Heth, G., Pratt, H. and Nevo, E. 1983. Photoperiodic effects on thermoregulation in a 'blind' subterranean mammal. J. Exp. Biol. 107: 59-64

Hart, L., Bennett, N.C., Malpaux, B., Chimimba, C.T. and Oosthuizen, M.K. 2004. The chronobiology of the Natal mole-rat, Cryptomys hottentus natalensis. Physiol. \& Behav. 82: 563-569.

Hart, L., O'Riain, M.J., Jarvis, J.U.M. and Bennett, N.C. 2006. Is the Cape Dune Mole-Rat, Bathyergus suillus (Rodentia: Bathyergidae), a Seasonal or Aseasonal Breeder? J. Mammal. 87: 1078-1085. 
Herbst, M., Jarvis, J. and Bennett, N. 2004. A field assessment of reproductive seasonality in the threatened wild Namaqua dune mole-rat (Bathyergus janetta). J. Zool. (Lond.). 263: 259-268.

Herbst, M. and Bennett, N.C. 2006. The burrow architecture and burrowing dynamics of the endangered Namaqua dune mole-rat, Bathyergus janetta (Rodentia: Bathyergidae). J. Zool. (Lond.). 270: 420-428.

Heth, G., Frankenberg, E., Raz, A. \& Nevo, E. (1987). Vibrational communication in subterranean mole rats (Spalax ehrenbergi). Behav. Ecol. Sociobiol. 21: 31-33.

Hrouzkova, E., Dvorakova, Jedlicka, P. and Sumbera, R. (2013). Sesimic communication in demon African mole rat Tachyorychtes daemon from Tanzania, J. Ethol. 31: 255-259.

Ims, R.A. 1990. The ecology and evolution of reproductive synchrony. Trends of Ecol. Evol. 5: $135-141$.

Isaac, J.L. and Johnson, C.N. 2003. Sexual dimorphism and synchrony of breeding: variation in polygyny potential among populations in the common brushtail possum, Trichosurus vulpecula. Behav. Ecol. 14: 818-822.

Jarvis, J.U.M. 1969. Some aspects of the biology of East African mole-rats. University of East Africa, Nairobi (unpublished $\mathrm{PhD}$ thesis).

Jarvis, J.U.M. and Sale, J.B. 1971. Burrowing and burrow patterns of East African mole-rats Tachyoryctes, Heliophobius and Heterocephalus. J. Zool. (Lond.). 163: 451-479.

Jarvis, J.U.M. and Bennett, N.C. 1991. Ecology and behaviour of the family Bathyergidae. In: J. U. M. Jarvis, R. D. Alexander. P.W. Sherman (ed.) The biology of the naked mole-rat. p 67-96. Princeton University Press, Princeton, NJ.

Katandukila, J.V., Faulkes, C.G., Chimimba, C.T. and Bennett, N.C. 2013. Reproduction in the East African root rat (Tachyoryctes splendens; Rodentia: Spalacidae) from Tanzania: the importance of rainfall. J. Zool. (Lond.). 291: 258-268.

Kennerly, T.E. Jr 1964. Micro-environmental conditions of the pocket gopher burrow. Texas J. Sci. 16: $395-441$.

Le Galliard, J., Remy, A., Ims, R.A. and Lambin, X. 2012. Patterns and processes of dispersal behavior in arvicoline rodents. Mol. Ecol. 21: 505-523 
Lilliefors, H.W. 1967. On the Kolmogorov-Smirnov Test for Normality with Mean and Variance Unknown. J. Am. Stat. Assoc. 62: 399-402.

Lovegrove, B.G. 1989. The cost of burrowing by the social mole-rats(Bathyergidae) Cryptomys damarensis and Heterocephalus glaber: the role of soil moisture. Physiol. Zool. 62: 449-469.

Lovy, M., Skliba, J. \& Sumbera, R. 2013. Spatial and Temporal Activity Patterns of the Free-Living Giant Mole-rat (Fukomys mechowii), the Largest Social Bathyergid. PloS one 8(1): e55357 (1-9).

McSweeney, C., New, M. \& Lizcano, G. 2010. UNDP climate change country profiles: Tanzania. Available: http://country-profiles.geog.ox.ac.uk/[Accessed 19 June 2016].

McSweeney, C., New, M., Lizcano, G. \& Lu, X. 2010. The UNDP climate change country profiles improving the accessibility of observed and projected climate information for studies of climate change in developing countries. Bulletin of the American Meteorological Society 91: 157-166.

Narins, P. M., Reichman, O., Jarvis, J. U.M. and Lewis, E. R. 1992. Seismic signal transmission between burrows of the Cape mole-rat, Georychus capensis. J. Comp. Physiol. A: Neur. Sens. Neur.Behav. Physiol. 170: 13-21.

Noack, T.H. 1894. Heliophobius emini n sp. Zool. Jahrb. Syst. 7: 559-562.

Oosthuizen, M.K., Cooper, H.M. and Bennett, N.C. 2003. Circadian rhythms of locomotoractivity in solitary and social species of African mole-rats (Family: Bathyergidae). J.Biol. Rhythms 18 (6): 481-490.

Oosthuizen, M.K. and Bennett, N.C. 2008. Seasonal variation in gonadal steroids of male and female in the Cape mole-rat (Georychus capensis): the potential for opportunistic breeding. Afr. Zool. 44: 117-122.

Oosthuizen and Bennett, N.C. 2015. The effect of ambient temperature on locomotor activity patterns in reproductive and non-reproductive female Damaraland mole-rats (Fukomys damarensis). J. Zool. (Lond.). 297:1-8.

Patzenhauerova, H., Bryja, J. and Šumbera, R. 2010. Kinship structure and mating system in a solitary subterranean rodent, the silvery mole-rat. Behav. Ecol. Sociobiol. 64: 757-767.

Pregitzer, K. S. and King, J.S. 2005. 10 Effects of soil temperature on nutrient uptake. Ecol. Stud. 181: 277-310. 
Rado, R., Levi, N., Hauser, H. \& Witcher, J. (1987). Seismic signalling as a means of communication in a subterranean mammal. Anim. Behav. 35: 1249-1251.

Rado, R., Wollberg, Z. and Terkel, J. 1992. Dispersal of young mole rats (Spalax ehrenbergi) from the natal burrow. J. Mammal. 73: 885-890.

Roper, T.J., Bennett, N.C., Conradt, L and Molteno, A.J. 2001. Environemntal conditionsin burrows of two species of African mole-rat, Georychus capensis and Cryptomys damarensis. J. Zool. (Lond.) 254: 101-107.

Scharff, A., Macholan, M., Zima, J. and Burda, H. 2001. A new karyotype of Heliophobius argenteocinereus (Rodentia, Bathyergidae) from Zambia with field notes on the species. Mamm. Biol. 66: 376-378.

Sichilima, A.M., Faulkes, C.G. and Bennett, N.C. 2008. Field evidence for seasonality of reproduction and colony size in the Afrotropical giant mole-rat Fukomys mechowii (Rodentia: Bathyergidae). Afr. Zool. 43: 144-149.

Sichilima A.M., Bennett, N.C. and Faulkes, C.G. 2011. Field evidence for colony size and aseasonality of breeding and in Ansell's mole-rat, Fukomys anselli (Rodentia: Bathyergidae). Afr. Zool. 46: 334-339.

Šklíba, J., Sumbera, R., Chitaukali, W. \& Burda, H. 2007. Determinents of daily activity patterns in a free-living afrotropical solitary subterranean rodent. J. Mammal. 88 (4): 10091016.

Šklíba, J. Lovy, M., Hrouzkova, E., Kott, O. Okrouhlik, J. \& Sumbera, R. 2014. Socail and Environmental Influences on Daily Activity in Free-Living Subterranean Rodents: The Case of a Eusocial Bathyergid. J. Biol. Rhythms 29 (3): 203-214.

Spinks, A.C, Van der Horst, G., and Bennett, N.C. 1997. Influence of breeding season and reproductive status on male reproductive characteristics in the common mole-rat, Cryptomys hottentotus hottentotus. J. Reprod. Fert. 109: 79-86.

Spinks, A.C, Bennett, N.C. and Jarvis, J.U.M. 1999. Regulation of reproduction in female common mole-rats (Cryptomys hottentotus hottentotus): the effects of breeding season and reproductive status. J. Zool. (Lond.). 248: 161-168. 
Šumbera, R., Burda, H. and Chitaukali, W.N. 2003. Reproductive biology of a solitary subterranean bathyergid rodent, The Silvery mole-rat (Heliophobius argenteocinereus). J. Mammal. 84: 278-287.

Šumbera, R., Šklíba, J. Elichlova, M., Chitaukali, W.N. \& Burda, H. 2008. Natural history and burrow system architecture of the silvery mole-rat from Brachystergia woodland. J. Zool. (Lond). 274: 77-84.

Tamura, K., Peterson, D., Peterson, N., Stecher, G., Nei, M., and Kumar, S. 2011. MEGA5: Molecular evolutionary genetics analysis using maximum likelihood, evolutionary distance, and maximum parsimony methods. Mol. Biol. Evol. 28: 2731-2739.

Van Der Walt, L., Bennett, N.C. and Schoeman, A.S. 2001. Reproductive suppression and pituitary sensitivity to exogenous GnRH in the highveld mole-rat, Cryptomys hottentotus pretoriae. J. Zool. (Lond.). 254: 177-184.

Van Rensburg, L. J., Bennett, N., Van der Merwe, M. and Schoeman, A. 2002. Seasonal reproduction in the highveld mole-rat, Cryptomys hottentotus pretoriae (Rodentia: Bathyergidae). Can. J. Zool. 80: 810-820.

Williams, L.R. and Cameron, G.N. 1984. Demography of Dispersal in Attwater's pocket gopher (Geomys attwateri). J. Mammal. 65: 67-75. 